\title{
Conception of Decision Support Tool for the Prevention and Detection of Type 2 Diabetes
}

\author{
Soumaya FELLAJI \\ Statistics Research Team \\ Faculty of Sciences and \\ Technologies (FST), Tangier, \\ Morocco
}

\author{
Abdellah AZMANI \\ Statistics Research Team \\ Faculty of Sciences and \\ Technologies (FST), Tangier, \\ Morocco
}

\author{
Abdelhadi AKHARIF \\ Statistics Research Team \\ Faculty of Sciences and \\ Technologies (FST), Tangier, \\ Morocco
}

\begin{abstract}
This paper describes the conception of a decision support tool which will, first, help healthy people to manage the risk to becoming diabetic and give them instructions to change their lifestyle in order to minimize it, and also, help diabetic people to avoid the risk of diabetes complications.
\end{abstract}

\section{General Terms}

Diabetes care, decision support system, telemedicine.

\section{Keywords}

Type 2 diabetes risk, decision support tool, fuzzy neural networks, Bayesian networks, constraints programming

\section{INTRODUCTION}

According to a report by the World Health Organization (WHO) made in 2006, chronic diseases are the leading cause of death of 17 million people worldwide each year. These chronic diseases include diabetes and visual or hearing impairments, cancer, heart disease and cardiovascular and chronic respiratory diseases [1].

These chronic diseases are due to some behavioral risk factors such as: smoking (this causes $9 \%$ of deaths), physical inactivity (the cause of $6 \%$ of deaths), poor diet and the harmful use of alcohol are responsible for $80 \%$ of coronary heart disease and cerebrovascular disease [1].

These chronic diseases are classified as non-communicable diseases (NCDs) and are responsible for the majority of deaths from these diseases. In the WHO statistics report 2012, cardiovascular diseases are responsible for $48 \%$ of NCD deaths, followed by cancers with a rate of $21 \%$ and chronic respiratory diseases represent $12 \%$ and finally diabetes is a direct cause of $3.5 \%$ of the deaths. Despite this value seems low, several complications can be caused as a result of diabetes diseases with a high rate like retinopathy (49\%), neuropathy (40\%), nephropathy (35\%) and cardiovascular disease (43\%) [1]. In addition, diabetes has several risk factors such as: Genetic predisposition, overweight, abdominal obesity, hypertension, unbalanced diet and sedentary. Furthermore, what is more dangerous is that diabetes is a silent disease and its symptoms appear only after a long silence for eight to ten years [2].

Considering the risk caused by different chronic diseases and especially this about diabetes, it is become essential to detect regularly the existence of this disease mainly while there are one or more risk factors.

To deal with this serious problem, a decision support tool will be developed to detect and prevent type 2 diabetes to minimize the risk of becoming diabetic and avoid the risk of diabetes complications for diabetic people. Therefore, this paper presents the proposed approach to concept the architecture of this tool.

\section{STATE OF THE ART}

Telemedicine (TM) is one of the areas experiencing a great development in which various research projects have been completed. It aims to satisfy a safe life for people with chronic diseases. It provides various tools to facilitate monitoring quality and collection of information about the monitored person's vital signs to analyze it and use it in order to evaluate and anticipate risks. Many projects have been created to meet the requirements of monitoring patients safely and reliably by monitoring their health and respecting the privacy of the patient.

In type 2 diabetes (T2D) field, there are four types of applications in TM which have been tested to improve the management of patients with this disease. These applications can be divided, first, into programs with educational goal, then, into monitoring applications which were performed by diabetes nurse educator by using telephone to contact patients and control their blood glucose. The third type consists of systems based on the transmission of blood glucose value and others vital signs which can present a risk factors for the management of diabetes, and finally, the use of the personal digital assistants ability and powerful to transmit data in real time and to communicate with a remote server [3].

The WellDoc system is appeared as a tool of coaching, comprising software hosted in a smartphone and in a web portal. Patient enters his data of auto-monitoring glycemic to smartphone application and receives, in real time, educative, comportemental and motivational messages. The web portal also includes a medical history and educational resources. A report in the form of a care plan is generated at regular intervals, for the patient and their general practitioner. The operation is supervised by specialized nursing in therapeutic education and able to intervene on the content of messages [4].

The "Sophia Programme", launched by the French health insurance fund for employees, is based on the individual risk level to treat patients. It aims to reduce the risk of diabetes complications and prevent the emergence of new 
complications. In case of patients at intermediate or high risk, telephone calls are offered by trained nurses [3].

Furthermore, after the success of DIABEO 1 for patients with type 1 diabetes, a version of this software has been developed for patients with T2D who have become insulin-dependent in order to propose an automatic adjustment of the dose of slowacting insulin in accordance with the medical prescription, and provide them daily coaching by advising on diet and physical activity [5]. In the same way, a study was conducted in France (TeleDiab-2) in 180 type 2 diabetic testing a system combining a titration help of basal insulin and a coaching by automated and personalized SMS [4].

Moreover, enormous efforts have been made in the search and the development of smart wearable systems for health monitoring due to the recent advances in micro- and nanotechnologies, miniaturization of sensors. In diabetes field, Medtronic's CGMS ${ }{ }$ and Guardian ${ }^{\circledR}$ are commercial products for continuous blood glucose-level monitoring systems. Another product called Abbott Diabetes care's FreeStyle Navigator ${ }^{\circledR}$ consists of a sensor placed on the skin a wireless transmitter attached to the sensor and a receiver, it provides data of blood glucose every minute. In addition, a biosensor connected to a micro-pump and coupled to a microdialysis system to construct a portable instrument able to measure blood glucose levels continuously, this commercial product is called Menarini Diagnostics’s Glucoday® [6].

Finally, the uses of web-based Personal Health Record and the Electronic Medical Records (EMRs) have been contributed to the improvement of diabetes care especially with the integration of intelligent records. These web-based programs include many features such as patients' access to EMRs, manual or automated feedback on blood glucose reading and other risk factors, educational information, etc. The Joint Asia Diabetes Evaluation Program (JADE) is one of web-based Personal Health Record which integrates different functions such as clinical decision, risk engine, self-management support and other many features to improve ambulatory diabetes care [7].

\section{PROPOSED APPROACH}

Regarding the exponential increase of type 2 diabetic rate around the world and the various factors and complications risk, it becomes very important to cope with this epidemic in order to improve people health quality and anticipate the risk of becoming diabetic. In comparison, given the different systems and research projects created, it can be concluded that there is a lack of intelligent systems which aim to minimize the risk for healthy people to becoming diabetic. Besides, not only metabolic and genetic factors can improve the risk of becoming diabetic but also behavioral and environmental factors like pollution and smoking. Nevertheless, these factors are not considered in these created systems.

For this purpose, the main goal is to create a decision support tool, able to take in consideration different factors influencing people health and to minimize risks by using and combining business intelligence techniques.

Thus, in the following, the need will be identified and validated, and then, a study of the system's feasibility will be done by determining the interaction between the system and its environmental elements so as to define the decision support tool architecture.

\subsection{Analyze the need}

\subsubsection{State the need}

To present the need of the proposed system, it is necessary to define why, how and for whom this system is created. The main goal consists of the creation of a decision support tool for the prevention and the detection of T2D. This tool intends different users (person who suffers from T2D or who wants to prevent the risk of becoming diabetic) and uses their vital signs to define their health statement. The following figure illustrates this need.

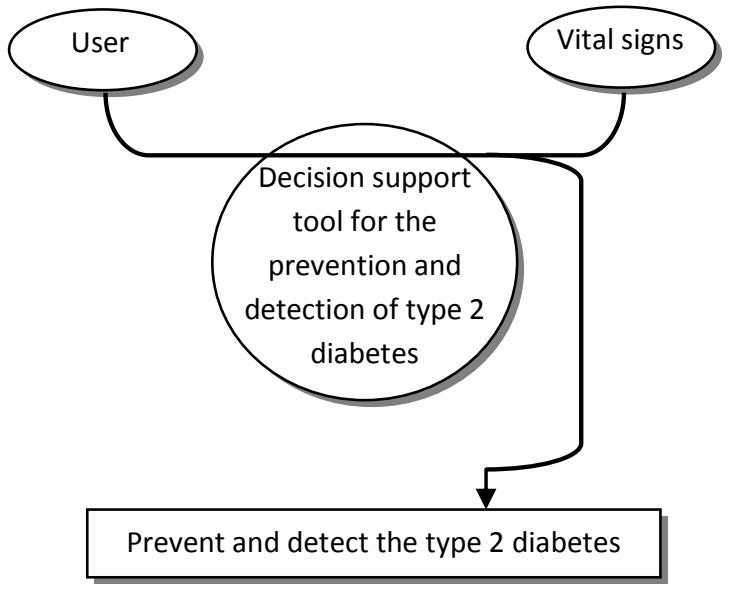

Fig 1: Illustration of proposed system's need

\subsubsection{Validate the need}

To validate the need of the proposed application, it is necessary to follow these steps:

Why is there this need?

- Goal: To prevent and detect type 2 diabetes.

\section{- Reasons:}

Because everyone has a risk of becoming diabetic.

ii.

iii. Because the percentage of people with diabetes is increasing exponentially.

i. Because diabetes is a silent disease

iv. Because diabetes has many risk factors.

$\mathrm{v}$

b. What could make it disappear or evolve? Because diabetes has several complications.

i. No one has a risk of becoming diabetic: impossible.

There isn't more increase in the percentage of people with diabetes: impossible (Given the current lifestyle).

iii. Diabetes occurs immediately when the person is affected: impossible.

iv. There is no risk factor causing diabetes: impossible.

Diabetes causes no complication: impossible. 


\subsection{Study the feasibility}

After analyzing the need, the first step is to investigate on the connections between the system and the external environment. This method defines the main functions (MF) in addition to the constraint functions (CF) (Table 1) to have an overview or a global view of the system.

To study the system's feasibility, it is necessary to define system's functions and its environmental elements (Fig 2).

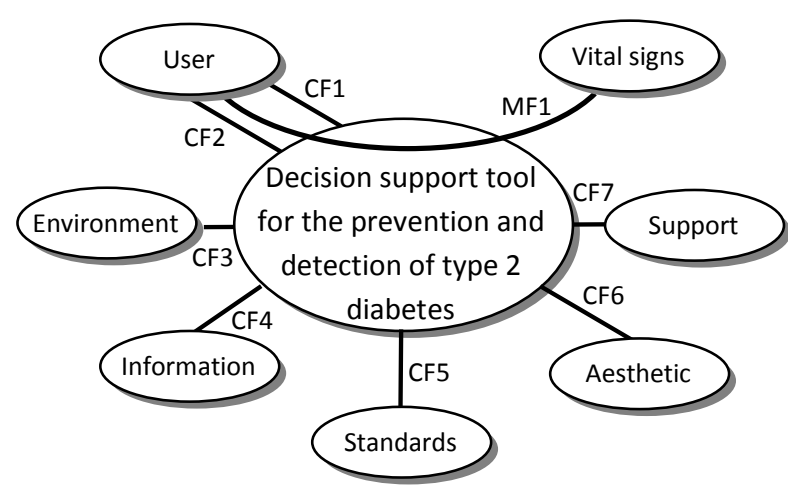

Fig 2: The interaction between the system and its environmental elements

Nevertheless, this diagram describes only the need and doesn't clarify which functions are received or generated by the main function.

\section{Table 1. List of system's functions}

\begin{tabular}{|l|l|}
\hline MF1 & Prevent and detect type 2 diabetes. \\
\hline CF1 & Be easily interrogated by the user. \\
\hline CF2 & $\begin{array}{l}\text { Be exploited by different users (patients and their } \\
\text { families, doctors, nurses, etc.). }\end{array}$ \\
\hline CF3 & $\begin{array}{l}\text { Consider environmental factors (pollution, smoking, } \\
\text { etc.). }\end{array}$ \\
\hline CF4 & $\begin{array}{l}\text { Respect the confidentiality of information from } \\
\text { others. }\end{array}$ \\
\hline CF5 & \begin{tabular}{l} 
To comply with international standards. \\
\hline CF6
\end{tabular} Be aesthetic. \\
\hline CF7 & Be adapted and installed in multiple media. \\
\hline
\end{tabular}

\subsection{System conception}

In this step, this paper presents the structured representation of the proposed system which helps to define the requirements and specify the functions in order to improve the design and the implementation of this system.

As it is shown in Fig 3, the proposed system takes in consideration two mains input data: monitored person's vital signs and environmental parameters. The main output data is the health state of monitored person besides the statement of his different vital signs and instructions to follow in diet, physical activity and lifestyle mode in addition to other recommendations to improve patient health state. This representation gives the function model which illustrates, in general, the process and the activity of this system's main function.

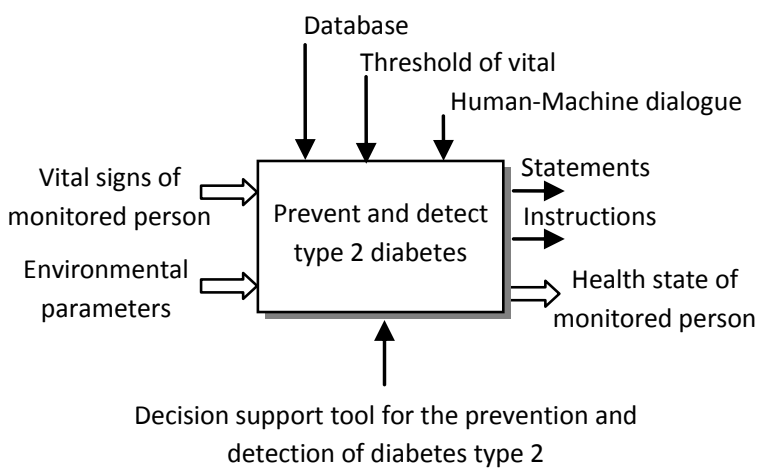

Fig 3: Structured representation of proposed system

\subsection{Technological solutions}

To have a detailed knowledge about the proposed system, it is necessary to know why and how the system's main function can be accomplished and how it can be decomposed into elementary functions to achieve the final solution (Fig 4).

The main function is "Prevent and detect type 2 diabetes", this function is decomposed into two elementary functions: "Acquire the values of vital signs" and "Analyze the values of vital signs".

\subsubsection{Sensors}

The first elementary function enables to gather information about the patient and the environmental parameter using sensors (personal and environmental sensors).

\subsubsection{Fuzzy neural networks}

The values gathered from sensors will be analyzed in order to identify the state of patient and then identify risk for healthy people by calculating the rate of factors risk and give them recommendations about their lifestyle, diet, physical activities and others recommendations concerning the patient environment. The achievement of these functionalities will be based on fuzzy neural network (FNN) which combines the powerful learning and calculating algorithms of neural networks technique with human knowledge presentation offered by fuzzy logic technique [8].

\subsubsection{Bayesian networks}

Bayesian networks (BN) are powerful diagnostic tools. In the medical field, they offer the possibility of taking into account both the expertise of the physician; statistical data on the incidence of diseases; and of course the specific observations of the patient. In case of sick people, the system will diagnose the disease for improving their treatment. To achieve this, BN technique will be used because of its power to model and represent knowledge to diagnosis problems [9]

\subsubsection{Constraint programming}

Constraint programming $(\mathrm{CP})$ is used to solve problems such as scheduling, planning, resource management, optimization of nonlinear systems, etc. Modeling problems uses variables 
and constraints between these variables [10]. Thus, this method can be used to planning interventions in emergency for people with type 2 diabetes.

\subsubsection{Alarm}

Finally, this system can help to anticipate crises and then alerting stakeholders by using alarm system.

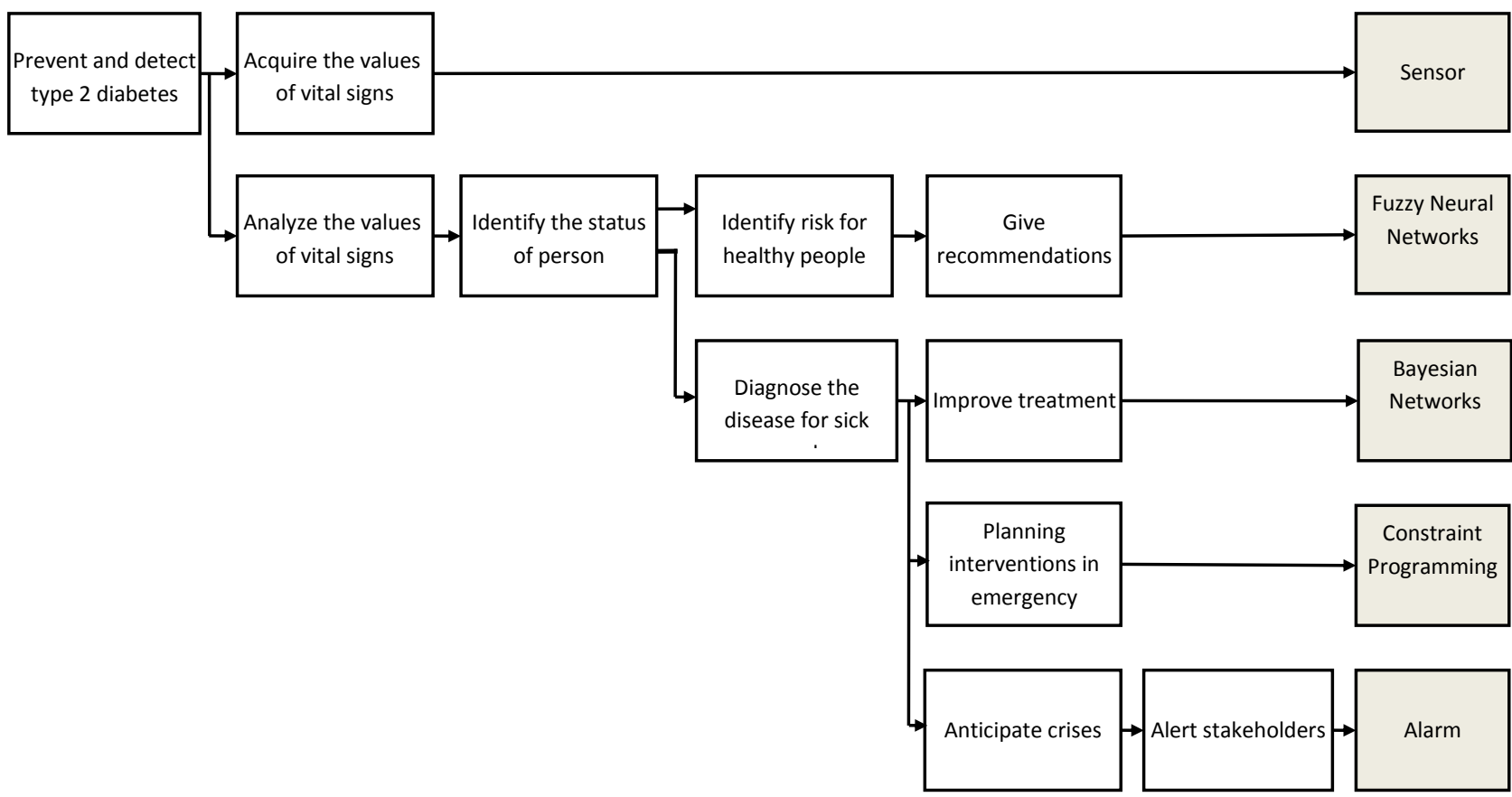

Fig 4: Functions tree for the technological solutions

\section{CONCLUSION AND PERSPECTIVES}

In this paper, a conception of a decision support tool for the prevention and detection of type 2 diabetes has been presented. It begins with the identification of needs until the detailed presentation of the proposed technology solutions design of such system. This work presents the preliminary step in order to help to construct the basics of this tool about the architecture of an intelligent system based on SAAS concept (Soft As A Service) which uses the presented functional structure (Fig 4). The future work consists of the experimentation of the FNN for identifying risk of T2D for healthy people and minimizes this risk to avoid its increase. In addition, others models will be created latter using $\mathrm{BN}$ and CP.

\section{REFERENCES}

[1] Statistiques sanitaires mondiales 2012. Genève: Organisation Mondiale de la Santé, 2012.

[2] M. Dr. Eric, La diététique du diabète. ÉDITIONS ALPEN, 2012.

[3] S. Franc, A. Daoudi, S. Mounier, B. Boucherie, D. Dardari, H. Laroye, B. Neraud, E. Requeda, L. Canipel, and G. Charpentier, "Telemedicine and diabetes: Achievements and prospects," Diabetes Metab., vol. 37, no. 6, pp. 463-476, Dec. 2011.
[4] P.-Y. Benhamou, M. Muller, S. Lablanche, and I. Debaty, "La télémédecine au service de la prise en charge des patients diabétiques : développements actuels et conditions du succès," Eur. Res. Telemed. Rech. Eur. En Télémédecine, vol. 2, no. 1, pp. 23-28, Mar. 2013

[5] S. Franc, A. Daoudi, B. Boucherie, C. Pechard, H. Laroye, S. Mounier, D. Dardari, L. Canipel, and G. Charpentier, "Telemedicine: Current status and prospects in type 2 diabetes," Eur. Res. Telemed. Rech. Eur. En Télémédecine, vol. 1, no. 2, pp. 76-79, Jun. 2012

[6] M. Chan, D. Estève, J.-Y. Fourniols, C. Escriba, and E. Campo, "Smart wearable systems: Current status and future challenges," Artif. Intell. Med., vol. 56, no. 3, pp. 137-156, Nov. 2012

[7] P. Y. Benhamou, "Improving diabetes management with electronic health records and patients' health records," Diabetes Metab., vol. 37, pp. S53-S56, 2011

[8] L. Wang and $\mathrm{X}$. Fu, Data mining with computational intelligence. Berlin; New York: Springer, 2005

[9] P. Naïm, P. H. Wuillemin, P. Leray, O. Pourret, and A. Becker, Réseaux bayésiens. Eyrolles, 2011

[10] R. B. Bachouch, "Pilotage opérationnel des structures d'hospitalisation à domicile," INSA de Lyon, 2010 